This Section of Epidemiology and Psychiatric Sciences regularly appears in each issue of the Journal to cover methodological aspects related to the design, conduct, reporting and interpretation of clinical and epidemiological studies. The aim of these Editorials is to help developing a more critical attitude towards research findings published in international literature, promoting original research projects with higher methodological standards, and implementing the most relevant results of research in every-day clinical practice.

Corrado Barbui, Section Editor and Michele Tansella, Editor EPS

\title{
Cluster randomised trials
}

\author{
C. Barbui* and A. Cipriani
}

Section of Psychiatry and Clinical Psychology, Department of Public Health and Community Medicine, University of Verona, Verona, Italy

\begin{abstract}
Although randomised controlled trials are the reference methodology to assess the effects of therapeutic interventions, for interventions that naturally occur in groups of individuals random allocation of participants may be inappropriate. In these cases, the unit of random allocation may be the group or cluster, rather than the individual. Clinical trials that randomly allocate groups or clusters of individuals are called cluster randomised trials. This article briefly presents the main implications of cluster randomisation with respect to the following methodological aspects: generalisability, concealment of allocation, comparability at baseline, blindness, loss of clusters and intra-class correlation.
\end{abstract}

Received 9 June 2011; Revised 10 June 2011; Accepted 18 June 2011

Key words: Bias, cluster, methods, randomised controlled trial.

Randomised controlled trials (RCTs) are considered the reference methodology to assess the beneficial and harmful effects of therapeutic interventions. The key aspect in RCTs is random allocation of individuals to competitive interventions. This procedure generates groups that are similar in terms of known and unknown characteristics (prognostic factors) (Cipriani et al. 2008). Similar groups of individuals who are exposed to different interventions can be compared at study end in terms of outcome measures, with the aim of establishing a relationship between the intervention under study and the outcome of interest.

In some circumstances, however, random allocation of individuals may be unsuitable. For example, it may be difficult to evaluate the efficacy of a set of clinical guidelines by randomising individuals to be treated according to the clinical guidelines or not. It would imply that each doctor should adopt and follow the

* Address for correspondence: Professor Corrado Barbui, Department of Public Health and Community Medicine, Section of Psychiatry and Clinical Psychology, University of Verona, Piazzale L.A. Scuro, 10-37134 Verona, Italy.

(Email: corrado.barbui@univr.it) set of clinical guidelines for those patients allocated to the intervention group, while simultaneously ignoring it for those allocated to the usual care group. Clearly, this approach is not only counterintuitive but also scientifically unsound, as doctors would inevitably be directly or indirectly influenced by the clinical guidelines even when treating the patients allocated to the control group, a phenomenon called 'contamination' across interventions. Other examples of interventions where random allocation of individuals may be inappropriate include organisational, educational or lifestyle interventions. In these cases, interventions naturally occur in groups of individuals, such as those followed by different doctors, medical practices, health care facilities and hospital wards. The groups may also be schools, villages or families. If interventions naturally occur in groups then the unit of random allocation may be the group or cluster, rather than the individual. Clinical trials that randomly allocate groups or clusters of individuals are called cluster randomised trials (Puffer et al. 2005).

Random allocation of clusters of individuals is graphically described in Fig. 1. A typical scenario involves groups of individuals followed by different 


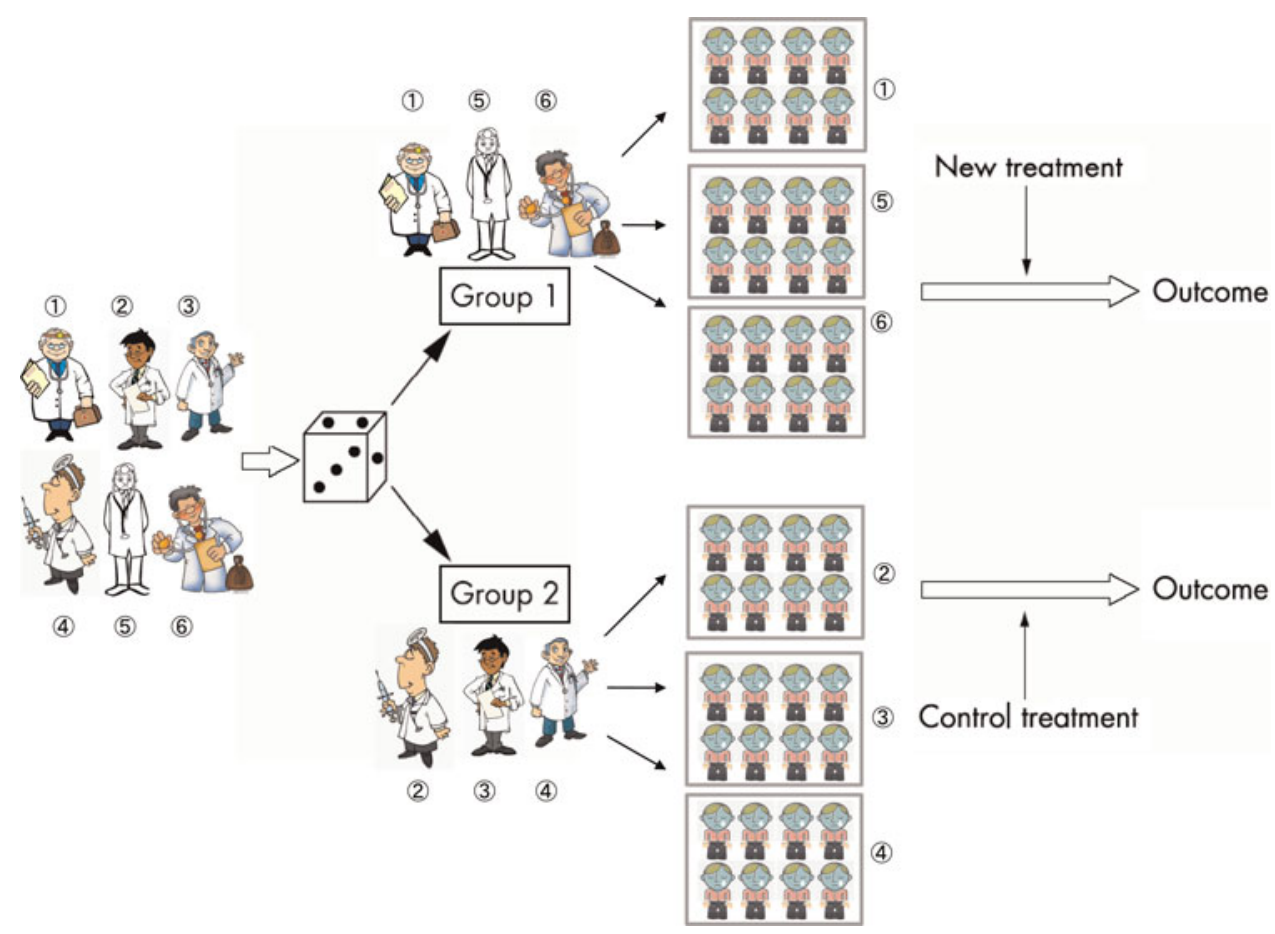

Fig. 1. Random allocation of clusters (groups) of individuals. (A colour version of this figure is available online at http://journals. cambridge.org/eps)

doctors. In the example there are six doctors who are randomly allocated to two different interventions (new treatment $v$. control treatment). Doctor No. 1, 5 and 6 are allocated to the new treatment arm and will therefore provide this intervention to all patients meeting the trial entry criteria (in this example, eight patients per doctor). These patients will constitute three clusters receiving the experimental intervention. Doctor No. 2, 3 and 4 are allocated to the control treatment arm and will therefore provide this intervention to all patients meeting the trial entry criteria (in this example, eight patients per doctor). These patients will constitute three clusters receiving the control intervention. At the end of the trial primary and secondary outcomes will be measured on all patients, which means 24 patients per treatment arm.

Intuitively, random allocation of clusters of patients is different from random allocation of individual participants, with implications in terms of study design, analysis and interpretation (Cipriani \& Barbui, 2010; Purgato et al. 2010). The main implications of cluster randomisation are briefly discussed below (Puffer et al. 2003; Hahn et al. 2005; Eldridge et al. 2008).

(1) Generalisability. In cluster trials, this aspect is related not only to the characteristics of the individuals included, as in individually randomised trials, but also to the characteristics of clusters. These may be judged by analysing the cluster entry criteria, which means, in the example reported above, information on number of doctors contacted, approached, recruited and analysed.

(2) Concealment of cluster allocation status. A major problem with cluster trials is that identification and recruitment of individuals occur after random allocation of clusters has been carried out. It is therefore possible that doctors enrol patients without being blind to the allocation status, and this might introduce a potential source of bias, as the knowledge of whether each cluster is an 'intervention' or 'control' cluster could affect the types of participants recruited. Hence, the potential for selection bias within clusters is particularly high (Eldridge et al. 2009). In some cluster trials, in order to minimise the risk of selection bias, all individuals within a cluster are included, or, alternatively, patient recruitment may be carried out by professionals who are masked to the cluster allocation status.

(3) Comparability at baseline. Random allocation of individuals aims at generating samples of individuals who are similar in group characteristics. This is not the case in cluster trials, as the unit of allocation is the cluster and not the individual. In cluster trials, there are two levels, the cluster and the individual, and random allocation should ensure comparability at baseline for clusters only. It is therefore of paramount importance to check the 
baseline summary information for both clusters (where comparability is expected) and individuals (where comparability may not be straightforward).

(4) Blindness. The characteristics of the intervention under study in cluster trials often do not allow blinding those delivering components of the intervention and, similarly, do not allow blinding individual participants receiving the intervention. Although this inability to blind is a distinctive feature of cluster trials, it is possible to assess outcomes blind to allocation status, for example by employing outcome assessors who are not involved in the conduct of the study and are masked to the allocated interventions.

(5) Loss of cluster. Loss of individual participants is a compelling issue in individually randomised trials. In cluster trials, in addition to this possibility, it is occasionally possible that complete clusters are lost, and have to be omitted from the analysis. In the example reported above, it is possible that one doctor is lost and, consequently, the corresponding cluster of eight patients is similarly lost. Clearly, just as for missing outcome data in individually randomised trials, this may lead to bias. In cluster trials, the issue is of high relevance, as groups of patients, rather than individual cases, may be lost.

(6) Intra-cluster correlation. In the example reported above, outcomes may be compared between the group of 24 patients who received the experimental intervention and the 24 patients who received the control intervention. One compelling issue, however, is that it cannot be assumed that observations on individuals belonging to the same cluster are independent, because it is possible that participants within any one cluster may share some known or unknown characteristics, and consequently may tend to respond in a similar manner. In cluster trials, observations on individuals tend to be correlated. This has implications in terms of sample size and analyses (Campbell et al. 2004c, 2005). Unfortunately, many of these studies are incorrectly analysed as though the unit of allocation had been the individual participants (Campbell et al. 2004b; Eldridge et al. 2004). This is often referred to as a 'unit-of-analysis error'. If outcomes are measured at the individual patient level, the analysis needs to be adjusted for potential clustering in the data. In contrast, if cluster trials are analysed as if individuals had been randomised, resulting $p$ values will be artificially small, resulting in false-positive conclusions that the intervention had an effect.

Reports of cluster randomised trials should include additional information to allow readers to interpret them accurately. The CONSORT statement has recently been extended to cluster randomised trials, offering this way a useful tool for investigators reporting the results of cluster trials and for readers of cluster trial reports (Campbell et al. 2004a). Readers may adopt the CONSORT statement to check the quality of reporting, making an informed judgement on the potential risk of bias associated with cluster randomised trials.

\section{References}

Campbell MK, Elbourne DR, Altman DG (2004a). CONSORT statement: extension to cluster randomised trials. British Medical Journal 328, 702-708.

Campbell MK, Grimshaw JM, Elbourne DR (2004b). Intracluster correlation coefficients in cluster randomized trials: empirical insights into how should they be reported. BMC Medical Research Methodology 4, 9.

Campbell MK, Thomson S, Ramsay CR, MacLennan GS, Grimshaw JM (2004c). Sample size calculator for cluster randomized trials. Computers in Biology and Medicine 34, 113-125.

Campbell MK, Fayers PM, Grimshaw JM (2005). Determinants of the intracluster correlation coefficient in cluster randomized trials: the case of implementation research. Clinical Trials 2, 99-107.

Cipriani A, Barbui C (2010). What is a clinical trial protocol? Epidemiologia e Psichiatria Sociale 19, 116-117.

Cipriani A, Nose M, Barbui C (2008). Allocation concealment and blinding in clinical trials. Epidemiologia e Psichiatria Sociale 17, 115-116.

Eldridge SM, Ashby D, Feder GS, Rudnicka AR, Ukoumunne OC (2004). Lessons for cluster randomized trials in the twenty-first century: a systematic review of trials in primary care. Clinical Trials 1, 80-90.

Eldridge S, Ashby D, Bennett C, Wakelin M, Feder G (2008). Internal and external validity of cluster randomised trials: systematic review of recent trials. British Medical Journal 336, 876-880.

Eldridge S, Kerry S, Torgerson DJ (2009). Bias in identifying and recruiting participants in cluster randomised trials: what can be done? British Medical Journal 339, b4006.

Hahn S, Puffer S, Torgerson DJ, Watson J (2005). Methodological bias in cluster randomised trials. BMC Medical Research Methodology 5, 10.

Puffer S, Torgerson D, Watson J (2003). Evidence for risk of bias in cluster randomised trials: review of recent trials published in three general medical journals. British Medical Journal 327, 785-789.

Puffer S, Torgerson DJ, Watson J (2005). Cluster randomized controlled trials. Journal of Evaluation in Clinical Practice 11, 479-483.

Purgato M, Barbui C, Cipriani A (2010). Assessing risk of bias in randomized controlled trials. Epidemiologia e Psichiatria Sociale 19, 296-297. 